\title{
Astronomical demonstration of superconducting bolometer arrays
}

Johannes G. Staguhn, Dominic J. Benford, Francois Pajot, Troy J. Ames, James A. Chervenak, et al.

Johannes G. Staguhn, Dominic J. Benford, Francois Pajot, Troy J. Ames, James A. Chervenak, Erich N. Grossman, Kent D. Irwin, Bruno Maffei, S. Harvey Moseley, Thomas G. Phillips, Carl D. Reintsema, Cyrille Rioux, Richard A. Shafer, George M. Voellmer, "Astronomical demonstration of superconducting bolometer arrays," Proc. SPIE 4855, Millimeter and Submillimeter Detectors for Astronomy, (17 February 2003); doi: 10.1117/12.459377

Event: Astronomical Telescopes and Instrumentation, 2002, Waikoloa, Hawai'i, United States 


\title{
Astronomical demonstration of superconducting bolometer arrays
}

\author{
Johannes Staguhn ${ }^{a^{*}}$, Dominic Benford ${ }^{a}$, Francois Pajot ${ }^{b}$, Troy Ames ${ }^{a}$, Jay Chervenak ${ }^{a}$, Erich
}

Grossman $^{c}$, Kent Irwin ${ }^{c}$, Bruno Maffei ${ }^{e}$, Harvey Moseley ${ }^{a}$, Thomas Phillips ${ }^{d}$, Carl Reintsema ${ }^{c}$, Cyrille Rioux ${ }^{b}$, Rick Shafer ${ }^{a}$, George Voellmer ${ }^{a}$

\author{
${ }^{a}$ NASA/Goddard Space Flight Center \\ ${ }^{b}$ Institut d'Astrophysique Spatiale, Universite Paris-Sud \\ ${ }^{c}$ NIST, Boulder \\ ${ }^{d}$ California Institute of Technology \\ ${ }^{e}$ Cardiff University
}

\begin{abstract}
We have built a prototype submillimeter spectrometer, FIBRE, which is based on a helium-cooled scanning Fabry-Perot and superconducting transition edge sensor bolometers (TES). SQUID multiplexers are used to read out the individual detector pixels. The spectral resolving power of the instrument is provided by the Fabry-Perot spectrometer. The outgoing light from the Fabry-Perot passes onto a low resolution grating for order sorting. A linear bolometer array consisting of 16 elements detects this dispersed light, capturing 5 orders simultaneously from one position on the sky. With tuning of the Fabry-Perot over one free spectral range, a spectrum covering $\Delta \lambda / \lambda=1 / 7$ at a resolution of $\sim 1 / 1200$ can be achieved. The spectral resolution is sufficient to resolve doppler broadened line emission from external galaxies. FIBRE operates in the $350 \mu \mathrm{m}$ and $450 \mu \mathrm{m}$ bands. These bands cover line emission from the important PDR tracers neutral carbon $[\mathrm{CI}]$ and carbon monoxide $\mathrm{CO}$.

The spectrometer was used at the Caltech Submillimeter Observatory to obtain the first ever astronomical observations using multiplexed arrays of superconducting transition edge bolometers.
\end{abstract}

Keywords: detectors, bolometers, multiplexers, sub-mm instruments, spectrometers

\section{INTRODUCTION}

The sub-mm and FIR wavelength regime still imposes a challenge on the design of efficient astronomical instruments, this is especially true for spectral line observations. The required large bandwidth combined with the low photon energies requires state of the art detector technology. There are two fundamentally different technologies available for observations at these frequencies: Heterodyne instruments (with mixers usually consisting of SIS Josephson Junctions at frequencies below or around $1 \mathrm{THz}$, and hot electron bolometers (HEB) at higher frequencies) provide excellent spectroscopic capabilities, but they suffer from backend bandwidth limitations and they have a limited noise performance at higher frequencies. The fundamental quantum noise of heterodyne instruments in units of Kelvin is $T=h v / k$ and therefore increases linearly with frequency. The quantum limit of heterodyne instruments, combined with the limited bandwidth of existing backends, imposes strong constraints on the sensitivity of these devices near and above $\mathrm{THz}$ frequencies. There is no such fundamental quantum limit for incoherent bolometer detectors, which are the second class of detectors working at these frequencies. These wavelength insensitive devices are only limited by thermal noise, allowing detector designs which achieve significantly lower noise temperatures than heterodyne instruments. Bolometers are therefore the best choice for sub-mm and FIR continuum observations. For spectral line observations, however, efficiency considerations often still favor heterodyne instruments for high spectral resolution applications. Consequently most of the first generation of bolometer instruments for the (sub-) mm regime, (e.g. SCUBA [1], SHARC [2], both operating at sub-mm wavelengths, and MAMBO [3] operating at $\mathrm{mm}$ wavelengths) are continuum devices. The FabryPerot Bolometer Interferometer Experiment, FIBRE, is an instrument designed to demonstrate a suite of advanced technologies, suitable for sensitive spectroscopic detection of far-infrared light. This includes superconducting transition edge sensor (TES) bolometers [4], multiplexed SQUID amplifiers [5], and a cryogenic Fabry-Perot interferometer for moderate resolution spectroscopy

*also SSAI; contact: staguhn@gsfc.nasa.gov; telephone: (301) 286-7840; Code 685, NASA / GSFC, Greenbelt, MD 20771, USA 
These components are being developed for the SOFIA imaging Fabry-Perot spectrometer SAFIRE [6] and for complement of ground-based instruments. FIBRE allows broad band $\Delta \lambda / \lambda=1 / 7$ low resolution $\Delta \lambda / \lambda=1 / 1200$ observations covering the $450 \mu \mathrm{m}$ and $350 \mu \mathrm{m}$ atmospherical windows. Fine structure lines of neutral carbon [CI] and mid-J rotational lines of $\mathrm{CO}$, all of which are important tracers of the interstellar medium and of the physical conditions in photo dissociation regions (PDR), are found in these bands.

\section{THE FIBRE INSTRUMENT}

FIBRE uses superconducting Transition Edge Sensor bolometers (TES), which were developed for observations ranging from X-ray to $\mathrm{mm}$ wavelength. TES have a number of advantages over semiconductor bolometers. Voltage biased TES are kept on the transition by "electrothermal feedback". This self regulating effect leads to the result that the combined electrical power due to the bias current plus the optical power from the incident photons is constant over a wide range of photon loads. The photon power can be measured by monitoring the bias current through the detector, which varies linearly with photon power over a wide range on the transition. The range of optical power over which a TES can be biased on the transition is determined by the difference between the transition temperature and the cold head temperature and the thermal link of the TES to this heat sink. A typical I-V curve for a FIBRE detector is shown in Fig. 3.

The TES in FIBRE have a transition temperature of $450 \mathrm{mK}$ and the detector package is cooled to $300 \mathrm{mK}$. TES devices, which are voltage biased and therefore operated in the electrothermal feedback regime, have a faster time constant than that of the intrinsic TES device [7]. Due to their low impedance $(\sim 100 \mathrm{~m} \Omega)$ TES are well matched to SQUID ammeters, which are used to sense the detector bias current. SQUID amplifiers provide a large noise margin and thus allow multiplexed readout. Staguhn et al. [8] show that the NIST designed SQUID multiplexers allow detector noise limited readout for our TES arrays. FIBRE features two 1 by 8 monolithic bolometer arrays consisting of $1 \mathrm{~mm}$ by $1 \mathrm{~mm}$ absorbers with a $300 \mu \mathrm{m}$ by $100 \mu \mathrm{m} \mathrm{Mo/Cu}$ bilayer TES. One of these detector arrays is shown in Fig.1. The small rectangles center bottom on the pixels are the Mo/Cu TES. Figure 2 shows an 8 channel NIST designed SQUID multiplexer with integrated "series array" pre-amplifier, consisting of $100 \mathrm{dc}$ SQUIDs in series.

The optical design uses a single Fabry-Perot etalon [9] followed by an order sorting grating. The grating is blazed to operate in one grating order where up to 6 of the Fabry-Perot orders are transmitted to the detector arrays with a dispersion of approximately $5 \mu \mathrm{m} / \mathrm{mm}$. In this manner, a spectrum consisting of several orders of the Fabry-Perot is collected instantaneously. The illumination for a closed packed array of 24 detector elements in FIBRE is shown in Fig. 4. In our observing run, the pixel positions in Fig. 4, labeled 1-2, 11-14, 23-24 were left blank. By stepping the FabryPerot over one free spectral range, a complete spectrum with a resolution of 1200 is accumulated over a bandwidth of almost $100 \mathrm{GHz}$. Figure 5 shows FIBRE's optical setup.

FIBRE's spectral resolution corresponds to a velocity resolution of about $250 \mathrm{~km} / \mathrm{s}$, which is well suited to resolve molecular line emission from external galaxies. The spectral resolving power was chosen, since the main scientific goal of FIBRE is the observation of emission lines from a variety of extragalactic sources. The large bandwidth, combined with the sensitivity, allows measurements of the underlying continuum emission. Both, the $\mathrm{CO}(\mathrm{J}=7-6)$ and $[\mathrm{CI}]\left({ }^{3} \mathrm{P}_{2}-{ }^{3} \mathrm{P}_{1}\right)$ spectral lines can be observed simultaneously in the $350 \mu \mathrm{m}$ atmospherical window.

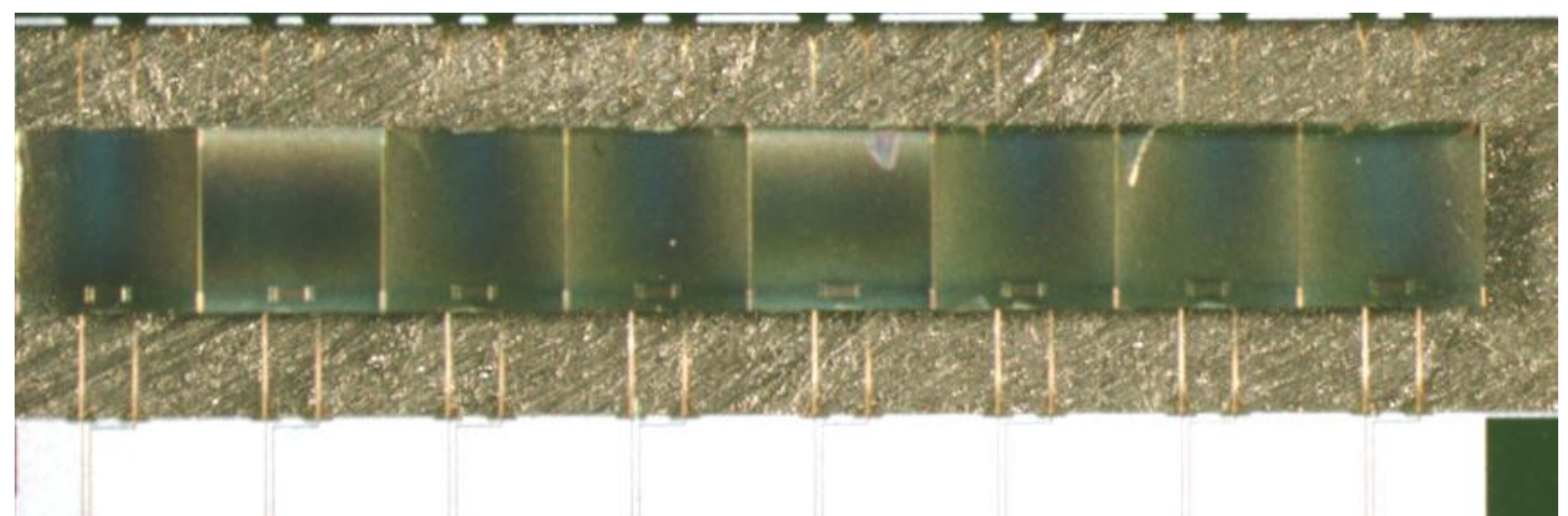

FIGURE 1. Photograph of a single 1 by 8 monolithic bolometer array. The $1 \mathrm{~mm}$ by $1 \mathrm{~mm}$ pixels are $1 \mu \mathrm{m}$ thick silicon membranes supported by 4 legs approximately $5 \mu \mathrm{m}$ wide. The TES is the small rectangle at the bottom center of each detector. 


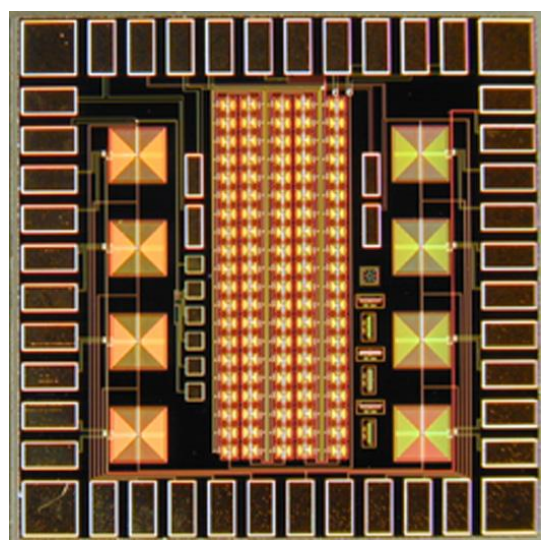

FIGURE 2. Photograph of the NIST designed 1 by 8 SQUID multiplexer used in FIBRE for the TES readout. The size of the chip is $\sim 6 \mathrm{~mm}$ by $6 \mathrm{~mm}$. The 8 square shaped devices are the input and the feedback coils with the 8 first stage SQUIDs in their center. The 100 squares in the center comprise the $2^{\text {nd }}$ stage "series array" consisting of 100 series SQUIDS which act as pre-amplifiers.

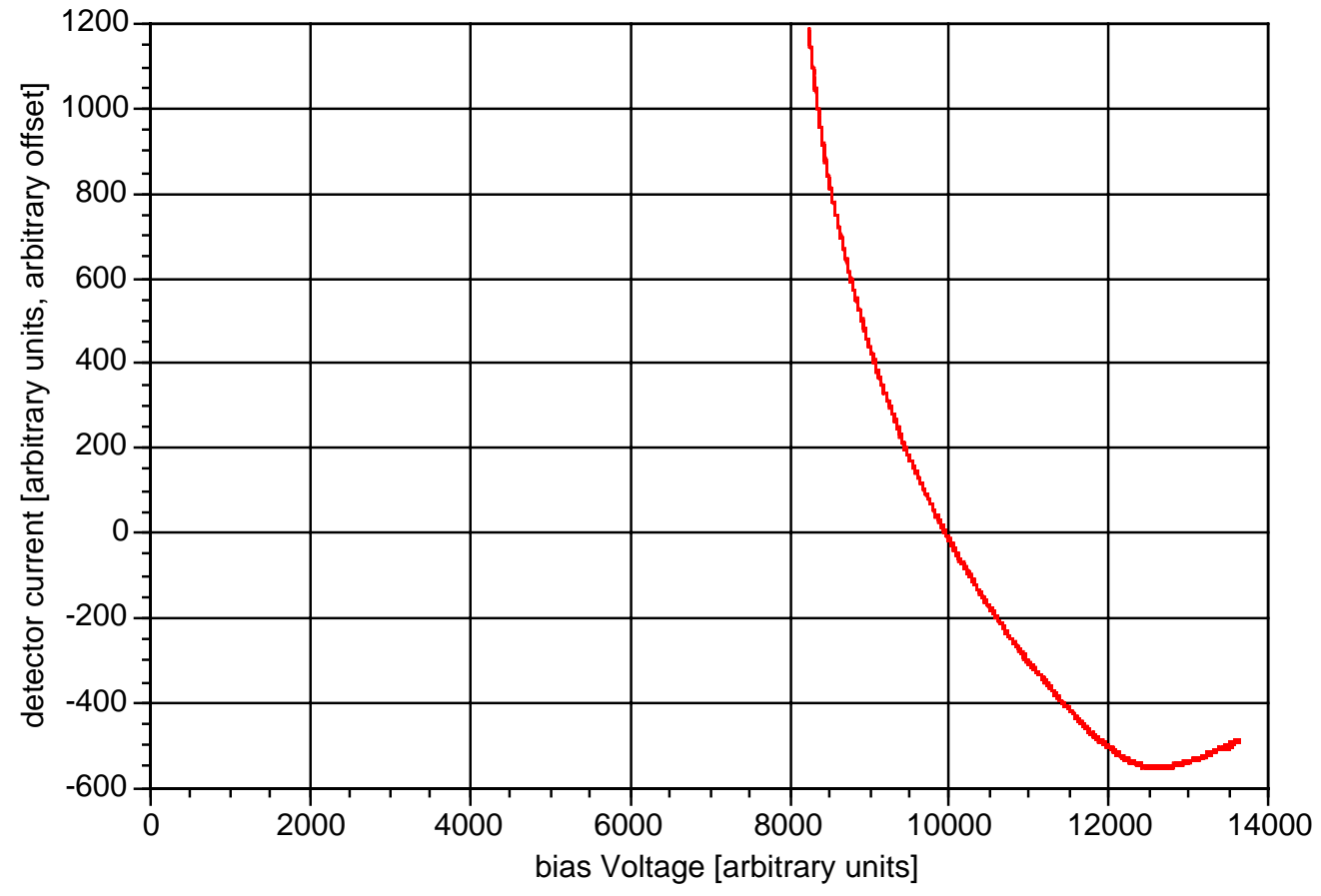

Figure 3. Typical I-V curve for a FIBRE TES detector. The falling part of the curve is the range on the transition where the dissipated electrical power is constant, i.e. it tracks a hyperbolic dependency of I over $\mathrm{U}$ with $\mathrm{I}=\mathrm{const} / \mathrm{U}$. The detector goes normal for the highest values of bias voltages. 


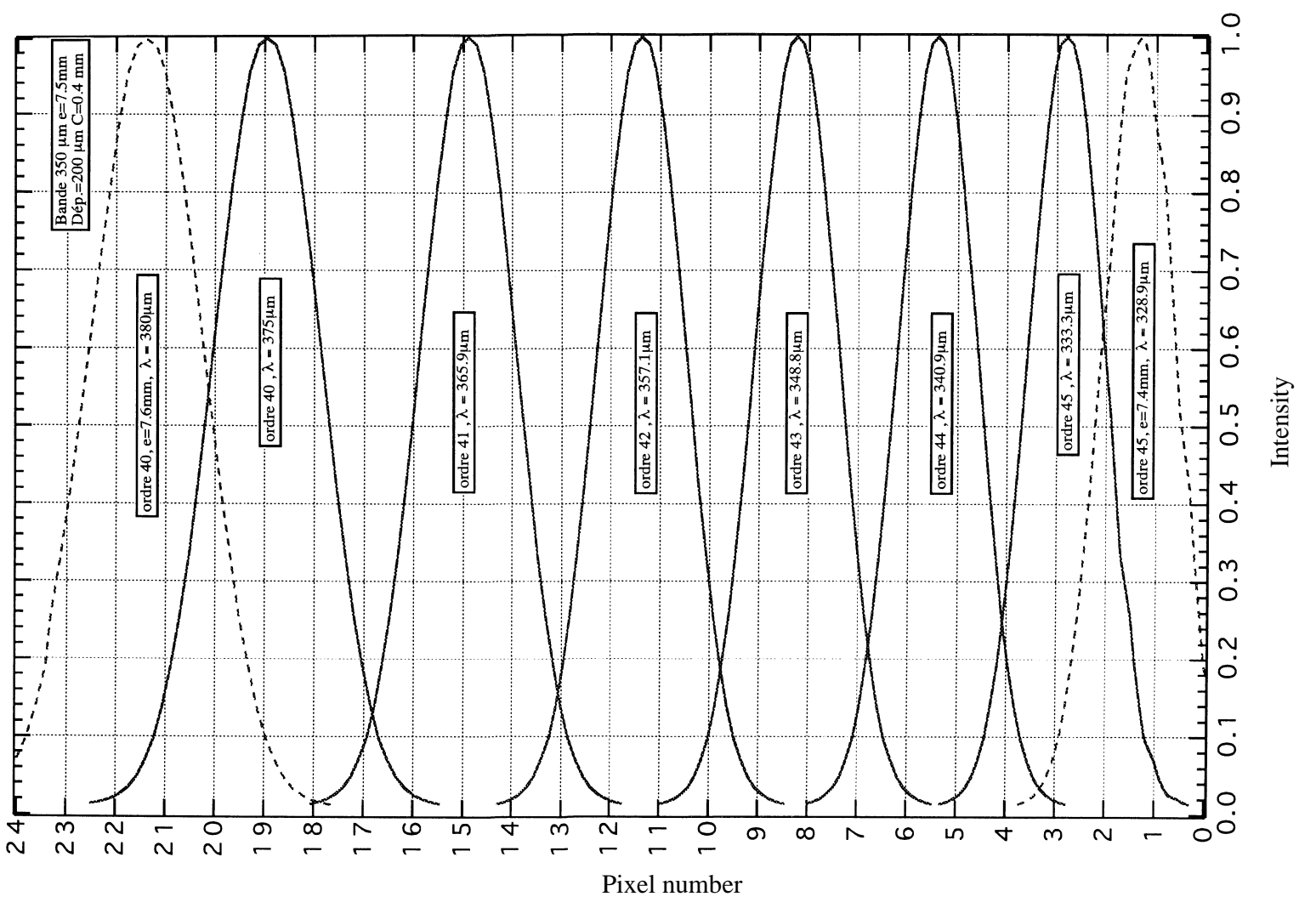

Figure 4. Illumination pattern of the detector array in FIBRE, here for a closed packed detector array with 24 elements. The actual 16 pixel coverage in the current FIBRE setup ranges from pixel number 2 through 9 and 14 through 21 on the figure shown here.

The strongest emission line in the $450 \mu \mathrm{m}$ atmospherical window is the $\mathrm{CO}$ ( $\mathrm{J}=6-5$ transition). With the large total bandwidth coverage of close to $100 \mathrm{GHz}$ per band, the observational bandwidth is only limited by the two atmospherical windows.

\section{OBSERVATIONS AND RESULTS}

FIBRE was first used at the Caltech Submillimeter Observatory (CSO) on Mauna Kea in Hawaii during an observing run in May/June 2001 (Fig. 6). The instrument was set up for observations in the atmospheric $350 \mu \mathrm{m}$ window. Initial test measurements with the black body loads and the CSO local oscillator verified the optical performance in multiplexed readout mode (Fig. 7). The spectral response of FIBRE was well in agreement with the predictions. Load measurements were used to derive the detector- as well as the optical- performance of the instrument on the telescope. The detector dark NEP was $\sim 3 \times 10^{-17} \mathrm{~W} / \mathrm{Hz}^{1 / 2}$. This corresponds to an rms noise of $40 \mathrm{mK} \mathrm{s}^{-1 / 2}$ at the entrance of the FIBRE dewar. The system temperature (outside the dewar) of FIBRE during the run corresponds to a radiometric noise temperature of $800 \mathrm{~K}$. The main reason for this relatively high value is FIBRE's low optical efficiency of 4\%, combined with the overillumination of the detectors, which further reduces the efficiency by a factor of roughly five. A spectral analysis of the un-chopped detector readout shows that the main noise contribution in the optical is low frequency $\sim 1 / \mathrm{f}$ noise which can be filtered by means of $4 \mathrm{~Hz}$ switching. One possible source for white noise radiation in the dark dewar was identified: During the observing run non-superconducting coils were used in the Fabry-Perot control motors. The coils turned out to significantly heat up the Fabry-Perot. We estimate that the emission from the motors could have added up to $1 \mathrm{pW}$ to the load on the detectors. After the run the bobbins were rewired with superconducting leads. The new motors produce no detectable heat dissipation. The improvements, especially the replacements of the Fabry-Perot motor wiring, will result in a better system noise performance in the future.

The 6 nights of observing time suffered from poor weather conditions. The best $350 \mu \mathrm{m}$ opacity we observed corresponds to $\tau_{350 \mu \mathrm{m}}=3$, typically the opacity was even worse with $\tau_{350 \mu \mathrm{m}} \sim 4$. Figure 8 (left) shows a skydip, which was observed with FIBRE. The opacities derived from FIBRE's skydips are well in agreement with those derived from 
the observatory's tau-meter. Despite the poor weather conditions, we managed to observe the limb of the moon. A scan over the edge of the moon is displayed in Fig. 8 (right). Each of the data points shown in the figure corresponds to an on source integration time of 3 seconds. These observations represent the first astronomical observations with TES bolometers in the sub-mm regime and they are the first astronomical observations ever with multiplexed readout of TES detectors.
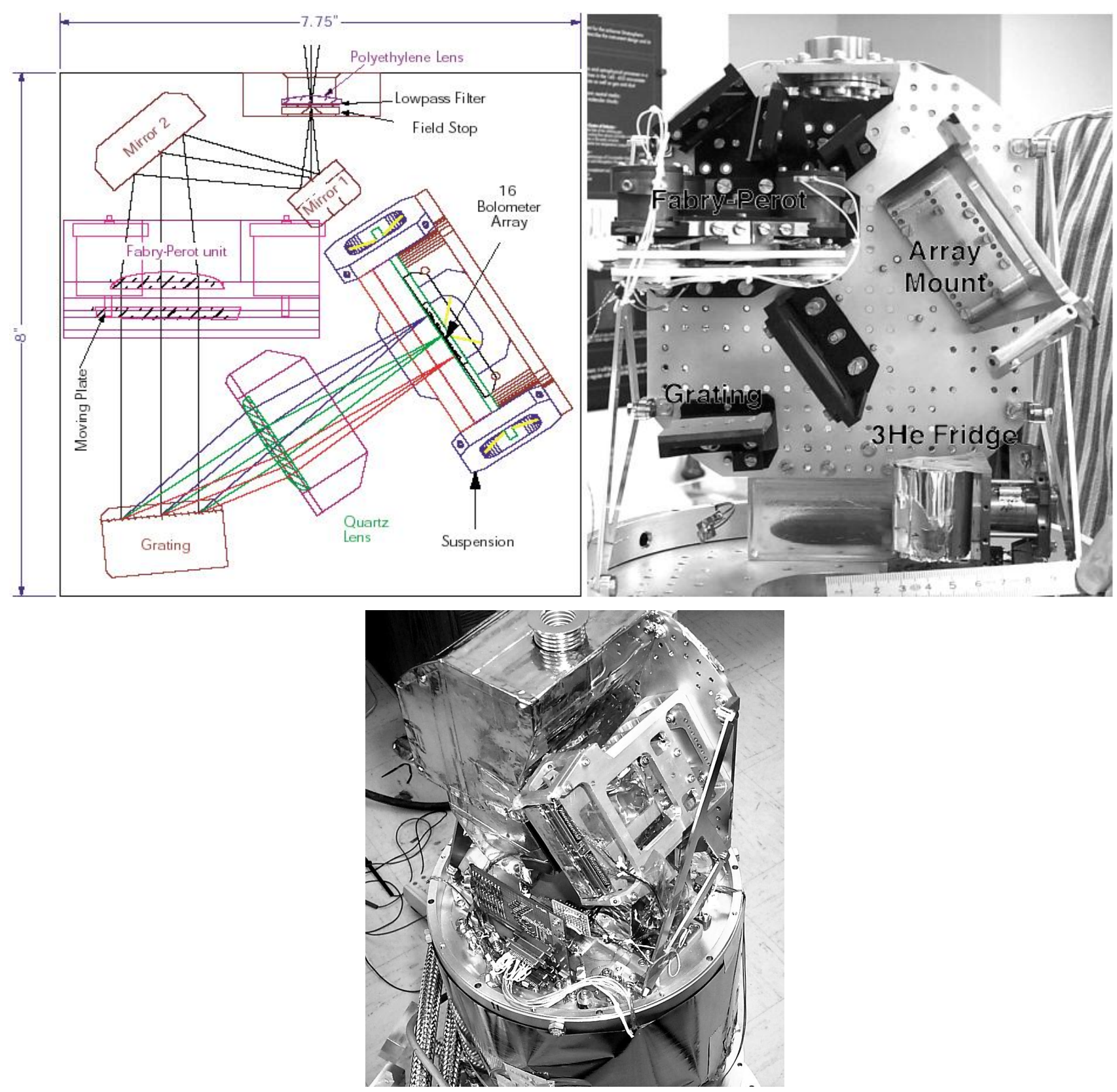

Figure 5. (top left) Diagram of the spectrometer optics, showing three orders being dispersed onto the bolometer array. (top right) Optics being assembled, with the detector array and baffles yet to be added, and (bottom) fully assembled. 


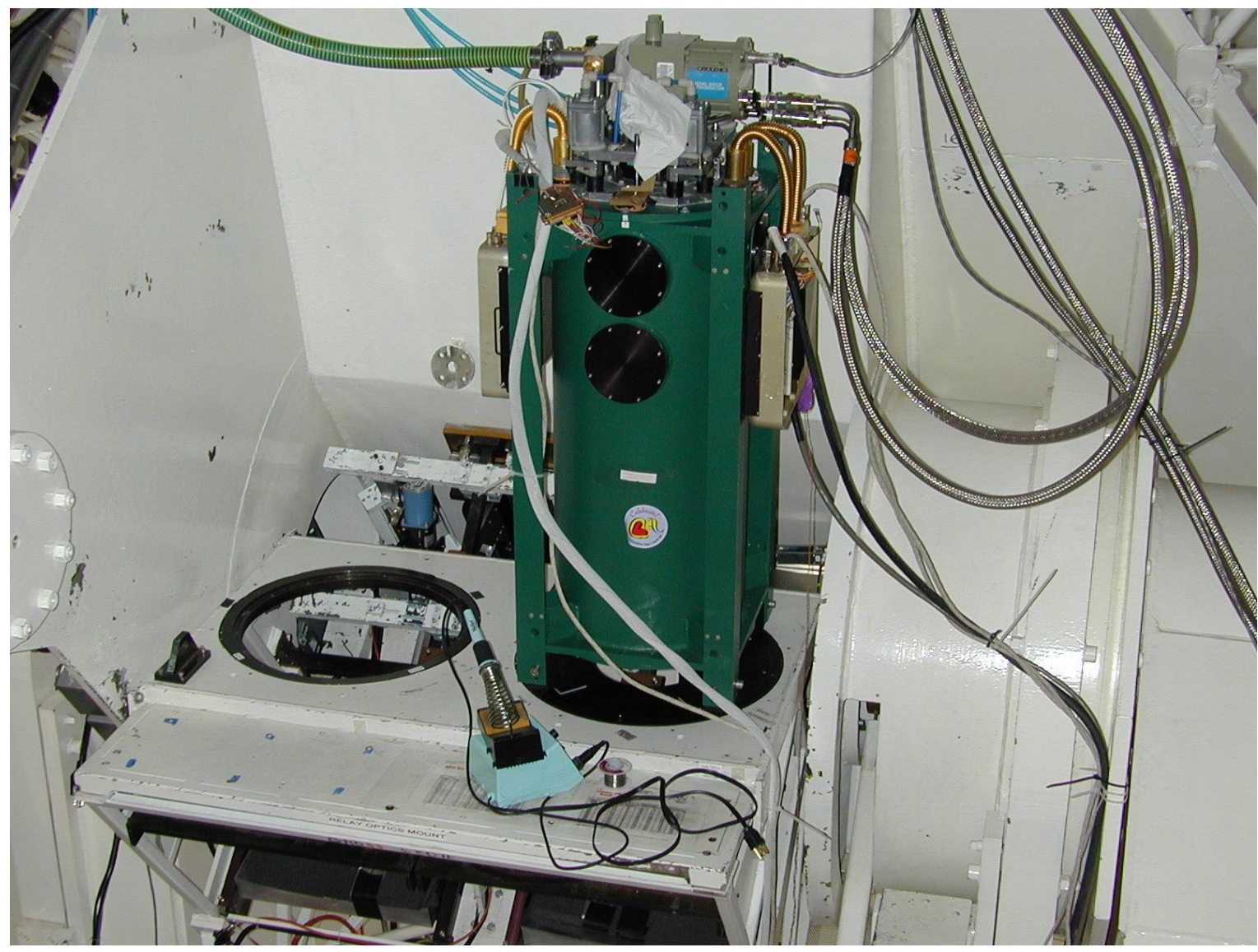

Figure 6. FIBRE in place on the Cassegrain relay focus of the Caltech Submillimeter Observatory on Mauna Kea, Hawai'i.

\section{CONCLUSION AND OUTLOOK}

The TES technology and the performance of our time domain SQUID multiplexers have been successfully demonstrated in sub-mm astronomical observations. We achieved first astronomical light ever with multiplexed readout of TES bolometers. The performance of FIBRE was close to the predicted values. In the meantime we improved the data acquisition system and fixed a problem with the Fabry-Perot motors. With these and other minor design changes we expect to be able to return to the CSO in the near future with a further improved system. Weather allowing, we plan to observe [CI] fine structure line emission and rotational $\mathrm{CO}$ lines from a number of extragalactic sources. These observations are aimed at gaining new insights into abundances and excitation of the neutral interstellar medium in a variety of galaxy types. Future FIR instruments, such as SAFIRE, which will fly on the SOFIA airborne observatory, will benefit from our experiences with FIBRE. Ultimately large two dimensional arrays of multiplexed low temperature transition edge sensors are a good candidate to be used in FIR space missions, aimed to close one of the largest "exploration space gaps" remaining in the electromagnetic spectrum.

\section{ACKNOWLEDGEMENTS}

We thank the staff of the Caltech Submillimeter Observatory for making the observations described here possible. Their support was crucial in the commissioning of FIBRE. We owe a debt of gratitude to many -too many to be named hereat NASA 's Goddard Space Flight Center for their contributions to the hardware and software that FIBRE uses. 

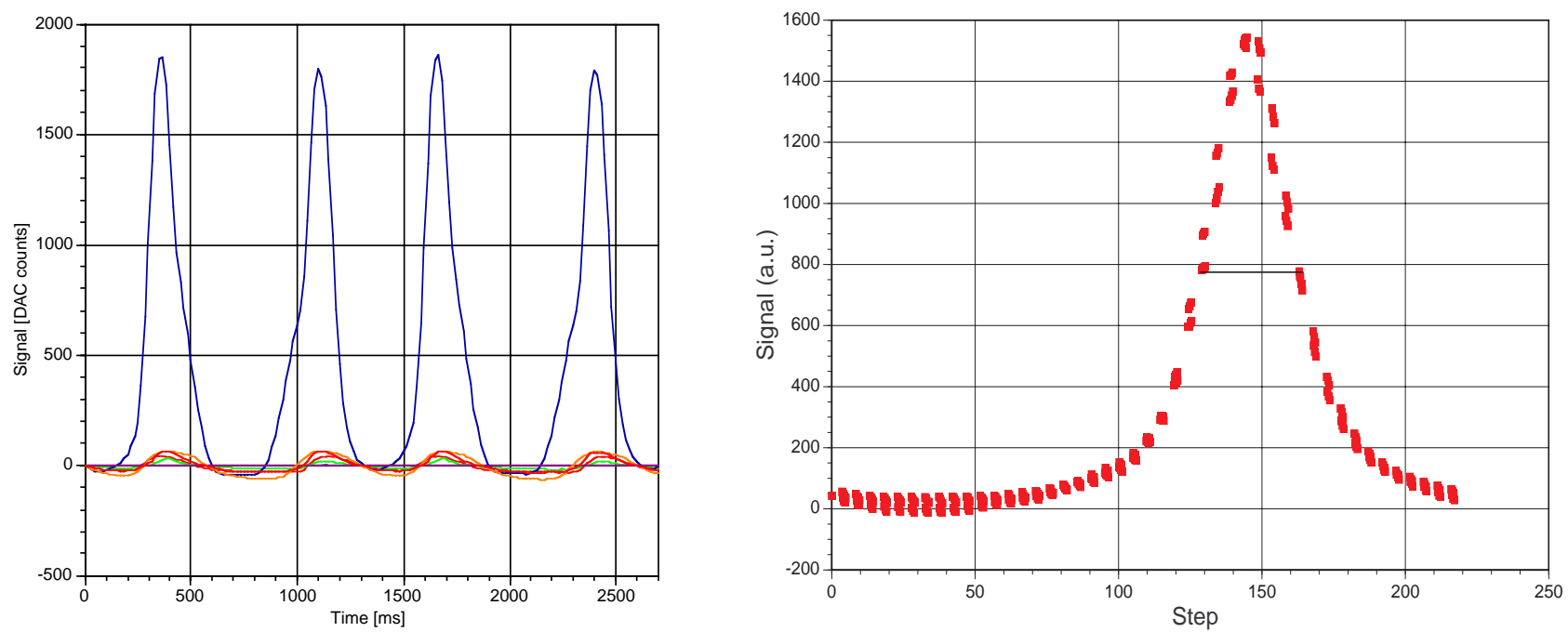

Figure 7. (left) The spectroscopic performance of FIBRE is demonstrated by the low response of adjacent channels. (right) Velocity calibration of a single channel with the CSO LO operating at $365 \mu \mathrm{m}(822 \mathrm{GHz})$. The resolving power is resolution is 1200 , corresponding to a velocity resolution of $250 \mathrm{~km} / \mathrm{s}$.
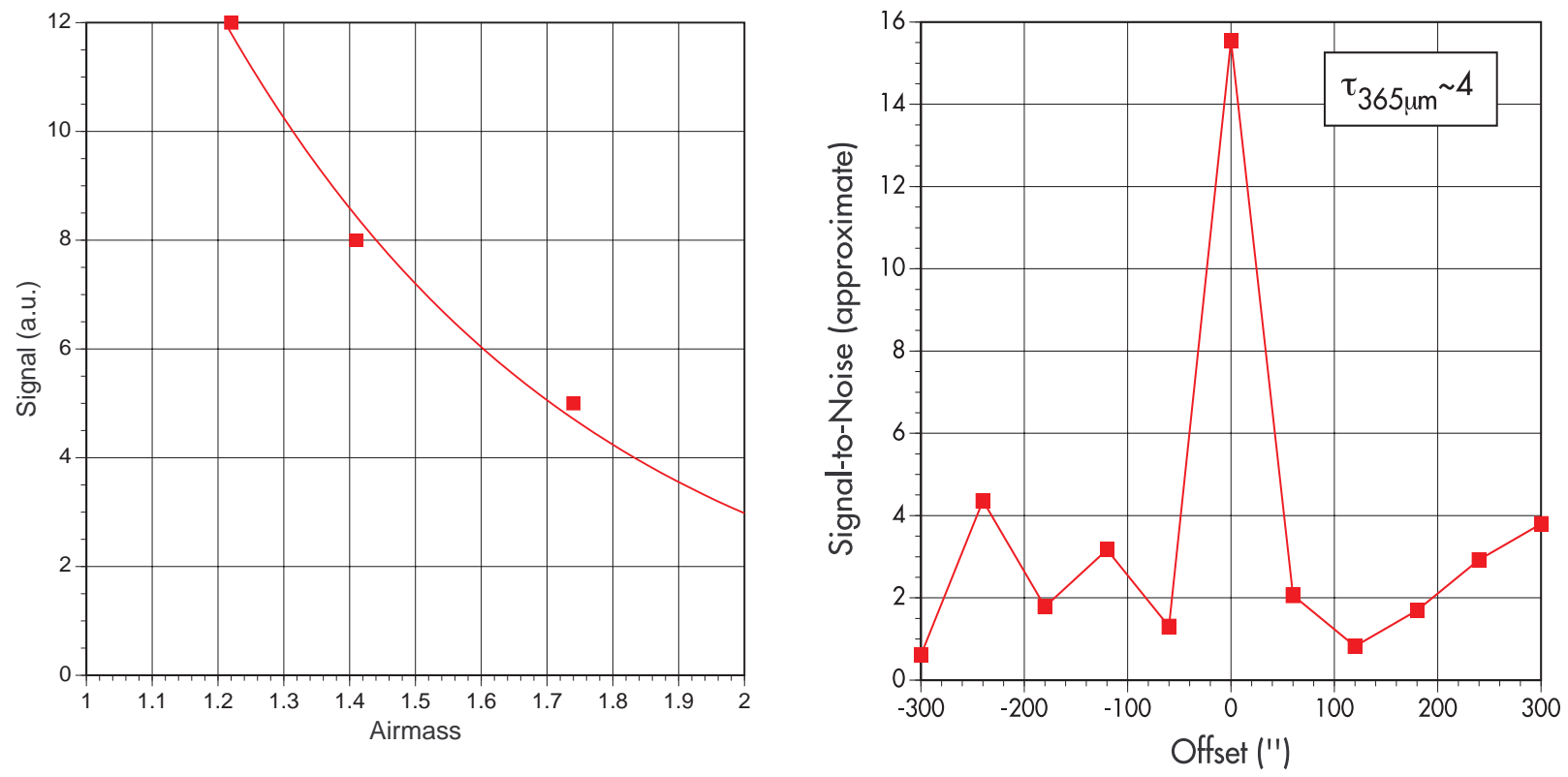

Figure 8. (left) FIBRE skydip yields $\tau(350 \mu \mathrm{m})=3.0$. (right) FIBRE detects the moon limb at $365 \mu \mathrm{m}$. Each data point corresponds to an on source integration for 3 seconds. 


\section{REFERENCES}

1. W.S. Holland, E.I. Robson, W.K.P., Gear, C.R. Cunningham, J.F. Lightfoot, T. Jenness, R.J. Ivison, J.A. Stevens, P.A.R. Ade, M.J. Griffin, W.D. Duncan, J.A. Murphy, D.A. Naylor, "SCUBA: a common-user submillimetre camera operating on theJames Clerk Maxwell Telescope", MNRAS, 303, pp.659-672, 1999

2. D.J. Benford, P. Cox, A. Omont, T.G. Phillips, \& R.G. Mc Mahon, "350 micron dust emission from highredshift objects", ApJL 518 , pp.65-68, 1999

3. E. Kreysa,.H.P. Gemünd, C.G.T. Haslam, L. Reichertz, E.E. Haller, J.W. Beeman, V. Hansen, A. Sievers, R. Zylka, "Bolometer array development at the Max-Planck Institut für Radioastronomie", SPIE, 3357, pp.319325, 1999

4. K.D. Irwin, "An application of electrothermal feedback for high resolution cryogenic particle detection", Appl. Phys. Lett., 66, pp.1998-2000, 1995

5. J.A. Chervenak, K.D. Irwin, E.N. Grossman, J.M. Martinis, C.D. Reintsema, M.E. Huber, "Superconducting multiplexer for arrays of transition edge sensors", Appl. Phys.Letters 74 (26),pp.4043-4045, 1999

6. R.A. Shafer. S.H. Moseley; P.A. Ade,.; D.J. Benford,.; G. Bjoraker, E. Dwek, D.A. Neufeld, F. Pajot, T.G. Phillips, G.J. Stacey, SPIE, 4014, p. 98-108, 2000

7. K.D. Irwin, G.C. Hilton, D.A. Wollman, J.M. Martinis, "Thermal-response time of superconducting transitionedge microcalorimeters", J. Appl. Phys., 83 (8), pp.3978-3985, 1998

8. J.G. Staguhn, C.A. Allen, D.J. Benford, J.A. Chervenak, M.M. Freund, S.A. Khan, A.S. Kutyrev, S.H. Moseley, R.A. Shafer, S. Deiker, E.N.Grossman, G.C. Hilton, K.D. Irwin, J.M. Martinis, S.W. Nam, D.A. Rudman, D.A. Wollman."TES Detector Noise Limited Readout Using SQUID Multiplexers", AIP Conf. Proc., 605, pp.321324, 2002.

9. B. Maffei, F. Pajot, T.G. Phillips, D. Benford, E. Caux, R. Gispert, N. Halverson, T. Hunter, G. Jegoudez, M. Josse, J.M. Lamarre, J.P. Lepeltier, H. Moseley, A. Origne, S. Petuchowsky, M. Rabottin, J.C. Renault, C. Rioux, G. Serabyn, N. Wang, “A medium resolution ground based submillimeter spectrometer", Infrared Phys. Technol, 35, 2/3, pp 321-329, 1994 